\title{
Pharmacological Profile of Ionotropic Glutamate Receptors in the Fly Neuro-Muscular Junction
}

\author{
I. M. Fedorova, L. G. Magazanik, and D. B. Tikhonov \\ Sechenov Institute of Evolutionary Physiology and Biochemistry, Russian Academy of Sciences, St. Petersburg; \\ e-mail: denistikhonov2002@yahoo.com
}

DOI: $10.1134 / \mathrm{S} 1990747809030209$

The action of series of pharmacological agents (agonists and competitive antagonists, allosteric modulators and channel blockers) was studied on the neuro-muscular junction of the fly Calliphora vicina larvae. The end-plate currents were evoked by nerve stimulation and recorded by conventional two-electrode voltage clamp. Properties of the ion channels were systematically analyzed using a series of 26 compounds, whose action was previously studied on mammalian NMDA and AMPA receptor channels. It was found that the structure-activity relationships of this set of channel blockers are similar for AMPA receptors and fly glutamate receptors. We also revealed two populations of fly receptors, which demonstrate about 20 different sensitivities to channel blockers.
The study of the glutamate-binding domain demonstrated unique pharmacological properties, which markedly differ from known mammalian receptors. Besides glutamate, fly receptors can be activated by kainate, quisqualate and domoate, but not AMPA and NMDA. Competitive antagonists of mammalian receptors were also ineffective against fly glutamate receptors. However, fly receptors, like certain AMPA receptors, were significantly potentiated by cyclothiazide. From this comparative study we came to the conclusion that glutamate-binding and channel-forming domains of ionotropic glutamate receptors evolved independently. 